\title{
Osteotomia triplanar no tratamento do escorregamento epifisário proximal do fêmur
}

\author{
Triplane osteotomy in the treatment of \\ slipped capital femoral epiphysis
}

Leandro Albuqueroue Lemgruber Kropf ${ }^{1}$, Celso Belfort Tizzi Júnior ${ }^{2}$, Bruno Tavares Rabello ${ }^{3}$, Fernando Pina Cabral ${ }^{4}$, Jorge Penedo ${ }^{5}$, Emílio Freitas ${ }^{5}$, Marco Bernardo Cury Fernandes ${ }^{5}$, Eduardo Regado Rinaldi ${ }^{5}$

\section{RESUMO}

Objetivo: Avaliar clínica e radiograficamente os resultados da osteotomia triplanar intertrocantérica no tratamento dos pacientes com escorregamento epifisário proximal do fêmur crônico. Métodos: Foram avaliados retrospectivamente 25 quadris, de 23 pacientes submetidos à osteotomia triplanar intertrocantérica no período de 1987 a 2003, que apresentavam escorregamento epifisário proximal do fêmur crônico (EEPF). Dezesseis pacientes eram do sexo masculino e sete do feminino,

* Trabalho realizado pelos Grupos de Ortopedia Pediátrica e de Reconstrução Articular do Instituto Nacional de Traumatologia e Ortopedia - INTO - Rio de Janeiro (RJ), Brasil.

1. Residente do Serviço de Residência em Ortopedia e Traumatologia do Instituto Nacional de Traumatologia e Ortopedia - INTO - Rio de Janeiro (RJ), Brasil.

2. Médico Ortopedista, Chefe do Centro da Criança e do Adolescente do Instituto Nacional de Traumatologia e Ortopedia - INTO - Rio de Janeiro (RJ), Brasil.

3. Residente do 4⿳⺈ ano do Grupo de Quadril do Instituto Nacional de Traumatologia e Ortopedia - INTO - Rio de Janeiro (RJ), Brasil.

4. Médico Ortopedista, Chefe do Grupo de Quadril do do Instituto Nacional de Traumatologia e Ortopedia - INTO - Rio de Janeiro (RJ), Brasil.

5. Médico Ortopedista do Grupo de Quadril do do Instituto Nacional de Traumatologia e Ortopedia - INTO - Rio de Janeiro (RJ), Brasil.

Endereço para correspondência: Bruno Rabello, Rua Tonelero, 380/ 302, Copacabana - 22030-000 - Rio de Janeiro (RJ), Brasil.

E-mail: brunotrabello@yahoo.com.br

Recebido em 15/1/07. Aprovado para publicação em 19/8/08. Copyright RBO2008 com média de idade de $\mathbf{1 4}$ anos e um mês, que tiveram seguimento clínico e radiológico médio de nove anos e três meses. A cirurgia foi realizada em pacientes com escorregamento moderado ou grave segundo a escala de Southwick (média de 57\%). $R e$ sultados: A classificação clínica e radiológica de Southwick foi utilizada na avaliação dos resultados e obtiveram-se $84 \%$ de resultados excelentes e bons nos critérios clínicos dor e função e $\mathbf{7 2 \%}$ de resultados excelentes e bons no critério radiológico, com avaliação final de $\mathbf{7 6 \%}$ de resultados excelentes e bons. Dos quatro resultados ruins verificados, dois apresentaram condrólise e dois evoluíram com degeneração articular. Não foi observado qualquer caso de necrose avascular. Conclusão: A osteotomia tridimensional intertrocantérica é boa opção de tratamento para os casos de EEPF com deslizamento maior de $30^{\circ}$, com resultados clínicos e radiográficos bons e excelentes em $76 \%$ dos pacientes.

Descritores - Osteotomia; Epífise deslocada/cirurgia; Resultado de tratamento

\section{ABSTRACT}

Objective: To make a clinical and radiographic evaluation of intertrochanteric triplane osteotomy in the treatment of patients with chronic slipped capital femoral epiphysis. Method: 25 hips of 23 patients with chronic slipped capital femoral epiphysis (EEPF) submitted to intertrochanteric triplane osteotomy from 1987 to 2003 were evaluated retrospectively. Sixteen of the patients were male, and seven, female, 
with a mean age of 14 years and one month, who had a mean clinical and radiological follow-up of nine years and three months. Surgery was performed in patients with moderate or severe slip according to the Sothwick scale (mean of 57 $)$. Results: The Southwick clinical and radiological classification was used to evaluate the results and results were $84 \%$ of excellent and good according to the clinical criteria of pain and function, and $72 \%$ of excellent and good results according to the radiological criteria, with a final evaluation of $76 \%$ of excellent and good results. Out of the four bad results achieved, two presented chondrolysis, and two developed joint degeneration. No case of avascular necrosis was seen. Conclusion: Intertrochanteric triplane osteotomy is a good treatment option for the cases of EEPF with a slip greater than $30^{\circ}$, yielding good and excellent clinical and radiographic results in $76 \%$ of the patients.

Keywords - Osteotomy; Epiphyses, slipped/surgery; Treatment outcome

\section{INTRODUÇÃO}

Nos últimos anos, a fusão da placa epifisária (epifisiodese), mediante utilização de um parafuso canulado único, tem sido relatada na literatura como o tratamento de escolha para o escorregamento epifisário proximal do fêmur (EEPF) com deslizamento leve e moderado. Autores têm demonstrado bons resultados, a longo prazo, inclusive em escorregamentos graves, demonstrando que a articulação do quadril tem grande potencial de remodelação ${ }^{(1-2)}$. Outros, porém, defendem que, em casos de deslizamento superior a $30^{\circ}$, submetidos à epifisiodese, a remodelação femoral não seria suficiente para restabelecer a biomecânica normal da articulação e, conseqüentemente, evitar o surgimento precoce da osteoartrose ${ }^{(3-4)}$. Estes pacientes deveriam ser submetidos a osteotomias femorais corretivas. A indicação destas osteotomias é ainda motivo de controvérsia na literatura, devido ao grande número de complicações relatadas ${ }^{(5)}$.

A osteotomia cervical é a que melhor realinha a epífise femoral, porque a correção é feita na topografia do próprio deslizamento; porém, existe, em relação a este procedimento, alto risco de evolução para necro- se avascular da cabeça femoral ${ }^{(6-10)}$. Visando diminuir estes riscos, outros métodos têm sido propostos, tais como osteotomia nas regiões subtrocantérica ${ }^{(11)}$, intertrocantérica $^{(4,12-13)}$ ou na base do colo ${ }^{(14)}$. Estas osteotomias extracapsulares podem reduzir as taxas de necrose asséptica, porém, são limitadas em relação ao grau de correção e produzem deformidades secundárias que poderão dificultar futuras cirurgias reconstrutivas $^{(6)}$.

O objetivo deste trabalho é avaliar clinicamente e radiograficamente 23 pacientes submetidos à osteotomia triplanar, em nível intertrocantérico femoral, para o tratamento do EEPF crônico moderado ou grave, com tempo médio de seguimento pós-operatório igual a nove anos e três meses.

\section{MÉTODOS}

Foram realizadas no Instituto Nacional de Traumato-Ortopedia/INTO/RJ, entre 1987 e 2003, osteotomias triplanares intertrocanterianas em 31 quadris de 29 pacientes portadores de EEPF crônica de moderada a grave, segundo a classificação de Southwick ${ }^{(11)}$ (tabela 1). Seis pacientes (seis quadris) foram excluídos: quatro que não retornaram à revisão ambulatorial, apesar de cinco convocações por telegrama e telefonema, e dois que não apresentavam dados nos prontuários que pudessem preencher o protocolo de inclusão no trabalho.

Foram avaliados, então, 25 quadris de 23 pacientes (tabela 2), com seguimento pós-operatório que variou de um ano a 17 anos e três meses (média de nove anos e três meses).

Dos 23 pacientes, 16 (69\%) eram do sexo masculino e sete $(31 \%)$, do feminino. A idade na data da cirurgia variou de 10 anos e sete meses a 17 anos e oito meses (média de 14 anos e um mês), sendo que a média entre os homens foi de 14 anos e nove meses e, entre as mulheres, de 12 anos e seis meses. Dos 25 quadris, 15 (60\%) eram do lado esquerdo e 10 (40\%) do lado direito. Dois pacientes, um do sexo masculino e outro do feminino, foram submetidos à cirurgia em ambos os quadris. Sete pacientes apresentavam a fise aberta na época da cirurgia, sendo realizada a epifisiodese no mesmo ato cirúrgico. 
TABELA 1

Classificação de Southwick

\begin{tabular}{|c|c|c|c|c|}
\hline & Excelente & Bom & Regular & Ruim \\
\hline Dor & $\begin{array}{l}\text { Sem dor ou os } \\
\text { pacientes ignoram a dor }\end{array}$ & $\begin{array}{l}\text { Dor após atividade } \\
\text { física }\end{array}$ & $\begin{array}{l}\text { Moderada, usualmente } \\
\text { com exercício, } \\
\text { ocasionalmente com } \\
\text { atividade normal }\end{array}$ & Grave e incapacitante \\
\hline Função & $\begin{array}{l}\text { Comparada ao lado } \\
\text { normal, pode fazer } \\
\text { trabalho pesado }\end{array}$ & $\begin{array}{l}\text { Ocasionalmente, pode } \\
\text { andar mais do que } \\
1,5 \mathrm{~km} \text {, trabalho } \\
\text { moderadamente pesado }\end{array}$ & $\begin{array}{l}\text { Cansa-se facilmente, } \\
\text { pode andar } 400 \mathrm{~m} \text {, pode } \\
\text { fazer trabalhos leves }\end{array}$ & $\begin{array}{l}\text { Não consegue andar } \\
400 \mathrm{~m} \text {, não consegue } \\
\text { fazer trabalhos leves }\end{array}$ \\
\hline Claudicação & $\begin{array}{l}\text { Sem claudicação, } \\
\text { Trendelenburg ausente }\end{array}$ & $\begin{array}{l}\text { Discreta claudicação, } \\
\text { Trendelenburg ausente }\end{array}$ & $\begin{array}{l}\text { Moderada claudicação, } \\
\text { Trendelenburg ausente }\end{array}$ & $\begin{array}{l}\text { Claudicação grave, } \\
\text { Trendelenburg presente }\end{array}$ \\
\hline $\begin{array}{l}\text { Arco de } \\
\text { movimento }\end{array}$ & $\begin{array}{l}\text { Diferença de até } 20^{\circ} \mathrm{em} \\
\text { relação ao lado normal }\end{array}$ & $\begin{array}{l}\text { Diferença de até } 40^{\circ} \mathrm{em} \\
\text { relação ao lado normal }\end{array}$ & $\begin{array}{l}\text { Diferença de até } 60^{\circ} \mathrm{em} \\
\text { relação ao lado normal }\end{array}$ & Mais de $60^{\circ}$ de diferença \\
\hline Radiologia & $\begin{array}{l}\text { Sem incongruência ou } \\
\text { estreitamento } \\
\text { significativo, Ângulo } \\
\text { cervicodiafisário até } \\
20^{\circ} \text { do normal }\end{array}$ & $\begin{array}{l}\text { Sem incongruência } \\
\text { significante. Moderado } \\
\text { estreitamento ou perda } \\
\text { da esfericidade, sem } \\
\text { alterações degenerativas } \\
\text { significantes, Ângulo de } \\
\text { até } 40^{\circ} \text { do normal }\end{array}$ & $\begin{array}{l}\text { Moderada incongruência, } \\
\text { moderada alteração } \\
\text { artrítica degenerativa, } \\
\text { sem necrose asséptica }\end{array}$ & $\begin{array}{l}\text { Grave incongruência e } \\
\text { alterações degenerativas, } \\
\text { ou necrose asséptica da } \\
\text { cabeça femoral }\end{array}$ \\
\hline
\end{tabular}

Fonte: J Bone Joint Surg Am. 1967;49(5):807-35.

TABELA 2

Avaliação da evolução dos casos operados

\begin{tabular}{|c|c|c|c|c|c|c|}
\hline Paciente & Idade & Sexo & Lado & Ângulo & Epifisiodese & $\begin{array}{c}\text { Tempo de } \\
\text { seguimento }\end{array}$ \\
\hline 1 & $13 a 8 m$ & $\mathrm{~F}$ & $\mathrm{D}$ & $62^{\circ}$ & $\mathrm{S}$ & 11a 7m \\
\hline 2 & 10a $11 \mathrm{~m}$ & $\mathrm{~F}$ & $\mathrm{D}$ & $60^{\circ}$ & $\mathrm{N}$ & 9a $10 \mathrm{~m}$ \\
\hline 3 & $12 \mathrm{a} 7 \mathrm{~m}$ & $\mathrm{~F}$ & $E$ & $58^{\circ}$ & $\mathrm{S}$ & 17a $3 \mathrm{~m}$ \\
\hline 4 & $12 \mathrm{a} 2 \mathrm{~m}$ & $\mathrm{~F}$ & $\mathrm{D}$ & $50^{\circ}$ & $\mathrm{N}$ & 13a $4 \mathrm{~m}$ \\
\hline 5 & $14 \mathrm{a} 9 \mathrm{~m}$ & $\mathrm{~F}$ & $E$ & $53^{\circ}$ & $\mathrm{N}$ & $8 a$ 6m \\
\hline 6 & $12 \mathrm{a} 9 \mathrm{~m}$ & $\mathrm{~F}$ & $\mathrm{D}$ & $48^{\circ}$ & $\mathrm{S}$ & $14 \mathrm{a} 1 \mathrm{~m}$ \\
\hline $7 *$ & $11 \mathrm{a} 1 \mathrm{~m}$ & $\mathrm{~F}$ & $\mathrm{D}$ & $64^{\circ}$ & $\mathrm{N}$ & $8 a$ \\
\hline $7 *$ & 11a $4 \mathrm{~m}$ & $\mathrm{~F}$ & $E$ & $68^{\circ}$ & $\mathrm{N}$ & $7 a 9 m$ \\
\hline 8 & 15a $2 \mathrm{~m}$ & $\mathrm{M}$ & $E$ & $50^{\circ}$ & $\mathrm{N}$ & $8 a$ 6m \\
\hline 9 & $14 \mathrm{a} 1 \mathrm{~m}$ & $\mathrm{M}$ & $\mathrm{D}$ & $52^{\circ}$ & $\mathrm{N}$ & $14 a$ \\
\hline 10 & 15a $2 \mathrm{~m}$ & $\mathrm{M}$ & $E$ & $70^{\circ}$ & $\mathrm{N}$ & 5a 3m \\
\hline 11 & $17 \mathrm{a} 8 \mathrm{~m}$ & $\mathrm{M}$ & $E$ & $93^{\circ}$ & $\mathrm{N}$ & $3 a 5 \mathrm{~m}$ \\
\hline 12 & $15 a$ 6m & $\mathrm{M}$ & $E$ & $44^{\circ}$ & $\mathrm{N}$ & $2 a 5 m$ \\
\hline 13 & 15a 10m & $\mathrm{M}$ & $\mathrm{D}$ & $66^{\circ}$ & $\mathrm{N}$ & $1 a$ \\
\hline 14 & $14 a 4 m$ & $\mathrm{M}$ & $E$ & $43^{\circ}$ & $\mathrm{N}$ & $14 \mathrm{a} 6 \mathrm{~m}$ \\
\hline 15 & $15 a 10 m$ & $\mathrm{M}$ & $\mathrm{D}$ & $48^{\circ}$ & $\mathrm{N}$ & $13 \mathrm{a} 9 \mathrm{~m}$ \\
\hline 16 & $16 a 5 m$ & $\mathrm{M}$ & $E$ & $56^{\circ}$ & $\mathrm{N}$ & $14 \mathrm{a} 3 \mathrm{~m}^{(1)}$ \\
\hline 17 & $14 a 4 m$ & $\mathrm{M}$ & $E$ & $72^{\circ}$ & $\mathrm{S}$ & $6 a 8 m$ \\
\hline 18 & $13 \mathrm{a} 1 \mathrm{~m}$ & $\mathrm{M}$ & $E$ & $51^{\circ}$ & $\mathrm{S}$ & $13 a 6 m$ \\
\hline $19 *$ & $16 a 3 m$ & $\mathrm{M}$ & $\mathrm{D}$ & $53^{\circ}$ & $\mathrm{N}$ & $8 a$ \\
\hline $19 *$ & $17 \mathrm{a} 2 \mathrm{~m}$ & $\mathrm{M}$ & $E$ & $61^{\circ}$ & $\mathrm{N}$ & $8 a 11 \mathrm{~m}$ \\
\hline 20 & $10 \mathrm{a} 7 \mathrm{~m}$ & $\mathrm{M}$ & $E$ & $49^{\circ}$ & $\mathrm{S}$ & $4 a 3 m$ \\
\hline 21 & $11 \mathrm{a} 5 \mathrm{~m}$ & $\mathrm{M}$ & $\mathrm{D}$ & $50^{\circ}$ & $\mathrm{S}$ & $8 a 3 m$ \\
\hline 22 & $14 \mathrm{a} 4 \mathrm{~m}$ & $\mathrm{M}$ & $E$ & $57^{\circ}$ & $\mathrm{N}$ & $6 a 3 m$ \\
\hline 23 & $14 a 5 m$ & $\mathrm{M}$ & $E$ & $48^{\circ}$ & $\mathrm{N}$ & $3 a 10 \mathrm{~m}$ \\
\hline
\end{tabular}

* - Pacientes com doença bilateral; (1) - Paciente submetido à ATO.

Legenda: a - anos, m- meses, M - Masculino, F - Feminino, D - Direito, E - Esquerdo, S - Sim, N - Não. 
O ângulo de deslizamento pré-operatório, avaliado na radiografia na incidência em Lauenstein (ângulo de Southwick), variou de $43^{\circ}$ a $93^{\circ}$, com média inicial de $57^{\circ}$.

\section{Planejamento pré-operatório}

Durante o planejamento pré-operatório foram analisadas radiografias em ântero-posterior e Lauenstein.

A anatomia radiológica normal consiste do ângulo cervicodiafisário em torno de $130^{\circ}$ e anteversão de $15^{\circ}$. Para que seja restabelecido o equilíbrio de forças no nível do centro de rotação da cabeça femoral após o escorregamento da epífise superior do fêmur, tentamos restaurar estas medidas, evitando, assim, sobrecarga mecânica que possa favorecer o desenvolvimento de processo degenerativo articular. A indicação cirúrgica foi feita quando se observou escorregamento superior a $30^{\circ}$ na incidência de perfil.

O gabarito para a osteotomia na face anterior é obtido subtraindo-se o ângulo cervicodiafisário do lado sadio ao do lado afetado, na radiografia em ânteroposterior. Já o gabarito para a osteotomia na face lateral é obtido por meio da diferença entre o ângulo de Southwick do lado afetado e a anteversão do lado normal, observadas na incidência de Lauenstein.

\section{Técnica cirúrgica}

Utilizamos o acesso lateral retilíneo expondo a face lateral da região intertrocantérica do fêmur. $\mathrm{O}$ próximo passo foi a osteotomia incompleta do grande trocanter, não descrita na técnica original, e foi realizada na grande maioria dos casos com objetivo de aliviar a tensão dos abdutores. A capsulotomia também foi realizada, bem como a tenotomia do tendão do psoas, com o intuito de diminuir a pressão sobre a cabeça femoral, reduzindo, assim, o risco de condrólise como complicação precoce.

A osteotomia intertrocantérica (de acordo com o planejamento pré-operatório), por meio de cunha de subtração, foi realizada corrigindo a deformidade em três planos. A osteotomia é fixada com uma placa lâmina de $130^{\circ}$ com cotovelo medializante, realizando-se de forma simultânea a epifisiodese em sete casos em que a fise ainda se encontrava aberta, utilizando-se um parafuso independente.
No pós-operatório, o paciente inicia marcha em 48 horas, após a retirada do dreno de sucção, com muletas em apoio parcial até a consolidação final da osteotomia.

\section{RESULTADOS}

Nossos resultados foram avaliados por meio da classificação descrita por Southwick ${ }^{(11)}$ em 1967, que utiliza critérios clínicos e radiográficos, classificando-os como excelente, bom, regular ou ruim (tabela 1).

Os critérios clínicos são: dor, função (avaliações subjetivas), claudicação e arco de movimento.

A avaliação radiológica foi realizada por meio de radiografias panorâmicas de bacia nas incidências em ântero-posterior e perfil da articulação acometida.

Os pacientes foram avaliados após tempo de cirurgia que variou de um ano a 17 anos e três meses (média de nove anos e três meses).

No critério dor, observamos que 15 pacientes não apresentavam dor e cinco apresentavam-na apenas após atividade física, caracterizando $80 \%$ de resultados bons e excelentes. Apenas três pacientes apresentavam dor moderada e dois revelavam dor intensa, inclusive ao repouso.

Quando avaliamos a função, observamos que 17 pacientes se apresentavam sem restrições funcionais e outros cinco apenas leves restrições, caracterizando pela classificação de Southwick $88 \%$ de resultados excelentes e bons ${ }^{(11)}$. Nesta mesma avaliação obtivemos $12 \%$ de resultados regulares e ruins.

$\mathrm{Na}$ avaliação conjunta dos critérios função e dor, ou seja, os critérios subjetivos, obtivemos $84 \%$ de excelentes e bons resultados e apenas $16 \%$ de resultados regulares e ruins.

No estudo visual da marcha, a claudicação não foi observada em 10 pacientes e em outros nove ela era apenas discreta, caracterizando $76 \%$ de resultados excelentes e bons. Nesta mesma avaliação, observamos que três pacientes apresentavam claudicação moderada e em três esta era grave, com sinal de Trendelenburg presente.

A análise do critério arco de movimento nos revelou os piores resultados dentre os critérios clínicos. Apenas dois pacientes $(8 \%)$ apresentaram arco de movi- 


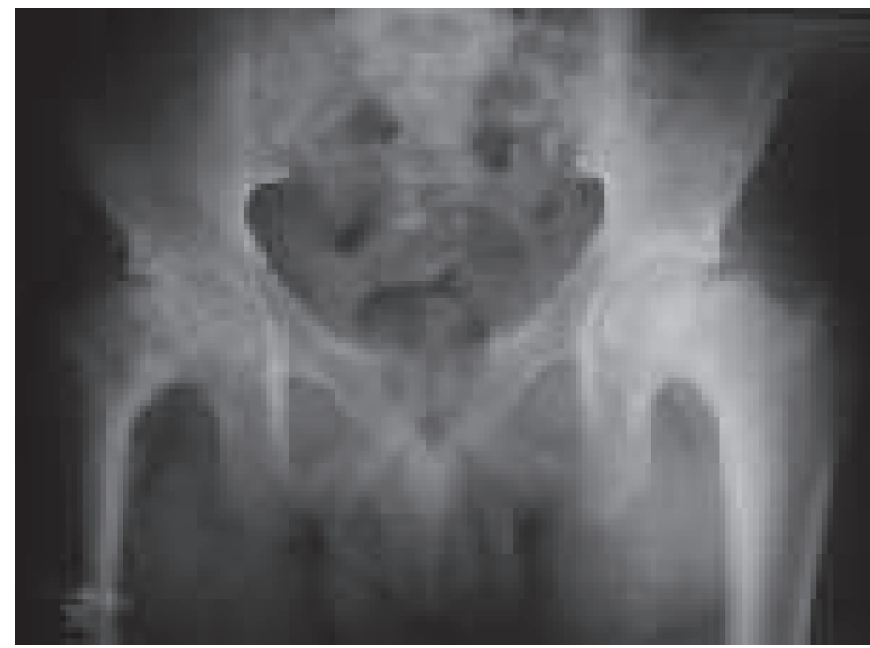

Figura 1 - Paciente com doença bilateral e ângulo de Southwick pré-operatório no lado direito de $53^{\circ}$ e lado esquerdo de $61^{\circ}$

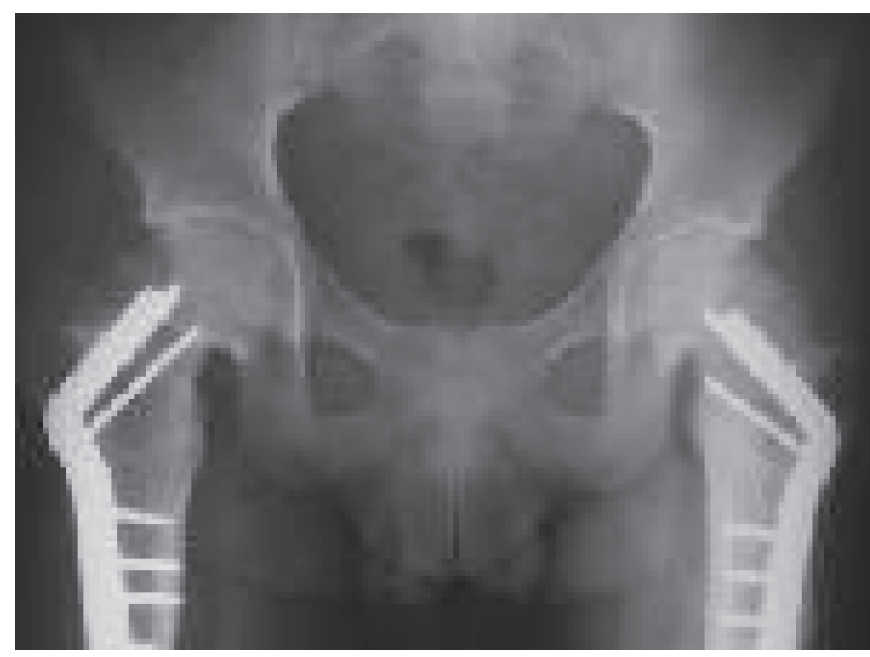

Figura 2 - Pós-operatório de osteotomia triplanar com seguimento de oito anos no lado direito e oito anos e 11 meses no lado esquerdo, com resultado final excelente no lado esquerdo e bom para o lado direito

mento excelente segundo a classificação de Southwick. Em 12 quadris (48\%) observamos bons resultados, caracterizando $56 \%$ de resultados excelentes e bons. Em cinco quadris identificamos resultados regulares e, em outros seis, ruins. Isto significou $44 \%$ de resultados regulares e ruins.

No estudo radiológico, classificamos nove quadris como excelentes, ou seja, sem incongruência significativa ou sinais degenerativos, e em outros nove quadris foram observadas apenas alterações degenerati-

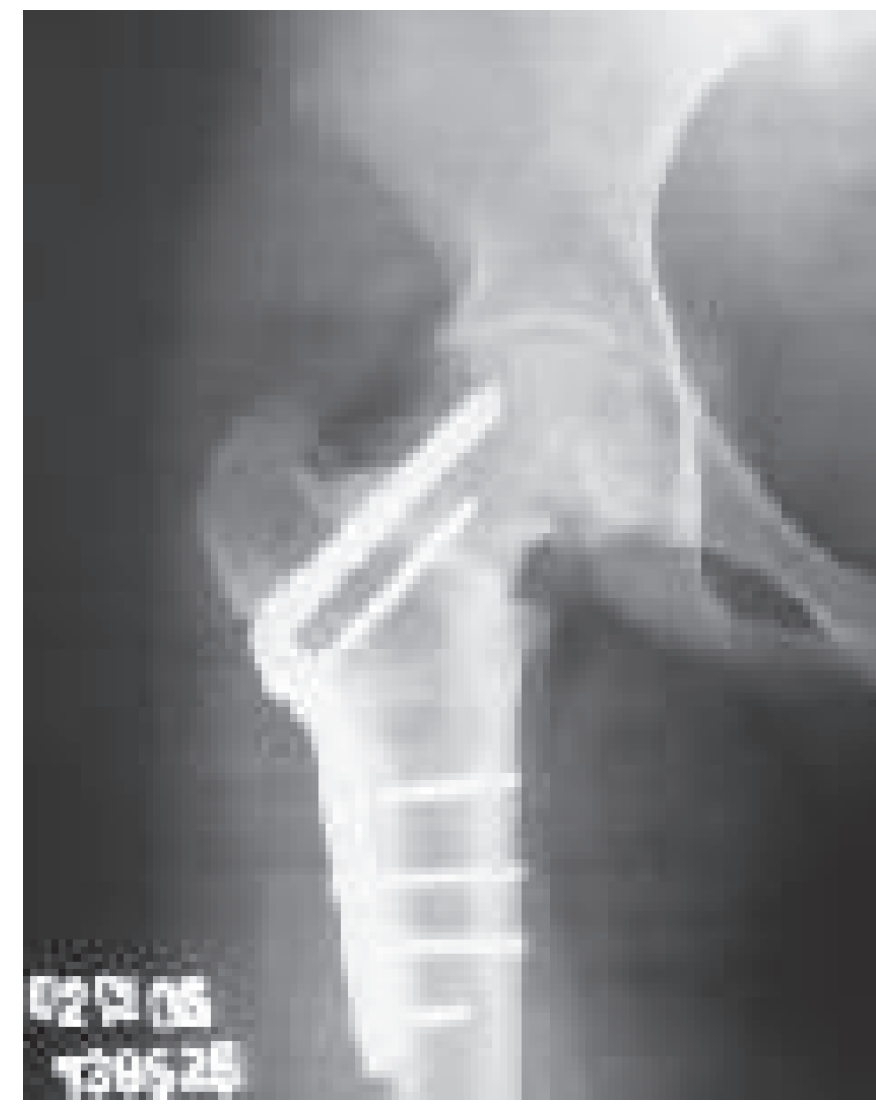

Figura 3 - Paciente masculino submetido a osteotomia triplanar com material de síntese intra-articular evoluindo com condrólise no pós-operatório e resultado final ruim

vas discretas, caracterizando um total de $76 \%$ de resultados excelentes e bons. Outros quatro quadris já se apresentavam com moderados sinais radiográficos de degeneração e três quadris foram classificados como radiologicamente ruins. Destes três, dois quadris apresentavam-se com sinais radiográficos de condrólise e o outro mostrava graves alterações degenerativas e incongruência articular. Não foi observada necrose avascular em qualquer dos quadris avaliados.

Fazendo uma avaliação final, somando todos os critérios avaliados pela classificação de Southwick ${ }^{(11)}$ e analisando-os conjuntamente, definimos 10 quadris (40\%) como apresentando resultados excelentes e nove (36\%), bons resultados, dois quadris ( $8 \%$ ) foram classificados como regulares e quatro $(16 \%)$ com resultados ruins. Dessa forma, obtivemos $76 \%$ de resultados excelentes e bons e $24 \%$ de resultados regulares e ruins (figuras 1, 2, 3). 


\section{DISCUSSÃO}

Na literatura não existe concordância em relação ao melhor método de tratamento para o EEPF com deslizamentos superiores ao de $30^{\mathrm{o}(5,14-17)}$. Porém, é consenso que, qual seja o tratamento escolhido, este é de difícil execução e sujeita a várias complicações.

O objetivo fundamental no tratamento desta patologia é a prevenção da osteoartrose precoce e isso só é possível se a biomecânica da articulação acometida for restabelecida ${ }^{(4)}$.

Alguns autores descreveram bons resultados finais com a fixação in situ com parafuso canulado único, mesmo em escorregamentos graves, justificando que o potencial de remodelação óssea do fêmur proximal seria suficiente para restabelecer uma boa função à articulação do quadril ${ }^{(1-2,18)}$, mas essa conduta não é respaldada em outros trabalhos ${ }^{(3,17)}$.

Bellemans, em série de 59 quadris fixados in situ, em 44 pacientes, com tempo de seguimento médio de 11,4 anos, obteve 53 quadris $(90 \%)$ classificados como excelentes ou bons, enfatizando, como justificativa dos resultados, a capacidade de remodelação do fêmur ${ }^{(1)}$.

Aronson et al, em série de 80 quadris fixados in situ, com acompanhamento médio de 3,3 anos, obtiveram $70 \%$ de resultados excelentes e bons ${ }^{(17)}$. Entretanto, quando dividiram os quadris pelo grau de deslizamento, observaram $86 \%$ de resultados excelentes e bons nos escorregamentos leves, $55 \%$ nos casos moderados e apenas $27 \%$ nos casos graves.

Outros autores acreditam que a única maneira de se restabelecer a biomecânica da articulação nos escorregamentos moderados e graves seria por meio da osteotomia corretiva ${ }^{(6-14)}$, que pode ser intracapsular ou extracapsular ${ }^{(19-20)}$.

A osteotomia do colo femoral, intracapsular, seria o único método capaz corrigir totalmente a deformidade, já que este procedimento é realizado no local em que o defeito ocorre, havendo também a vantagem de não promover alteração da anatomia do fêmur, o que não prejudicaria cirurgias reconstrutivas no futuro. Além disso, a maioria das publicações sobre osteotomias do colo do fêmur afirma que quanto maior o grau de deslizamento, maior a incidência de necrose avas- cular; logo, em pacientes com deslizamento maior do que $30^{\circ}$, o índice de complicações poderia ser eleva$\mathrm{do}^{(6,8-9)}$.

Em série de 42 osteotomias cuneiformes do colo femoral, Fish obteve apenas 2,4\% de necrose avascular, defendendo que esta osteotomia pode ser realizada, com poucas complicações, por cirurgiões com experiência no método ${ }^{(7)}$; porém, Gage et al relatam 72 osteotomias do colo femoral, com 37,6\% de condrólise e $28,5 \%$ de necrose, o que os levou a abandonar a técnica, devido à alta incidência de complicações ${ }^{(8)}$. Hägglund et al, em série de 33 osteotomias cervicais, com seguimento médio de 28 anos, também obtiveram altos índices de necrose $(24,2 \%)$, além de $6 \%$ de condrólise e $57,5 \%$ de artrose secundária( ${ }^{(9)}$.

No nosso trabalho foram avaliados 23 pacientes ( 25 quadris) submetidos à osteotomia triplanar intertrocanteriana, com tempo médio de seguimento de nove anos e três meses; comparando-se os critérios clínicos subjetivos com os objetivos, observamos que não ocorreu concordância entre eles, pois obtivemos $84 \%$ de resultados excelentes e bons avaliando-se dor e função, e $66 \%$ de resultados excelentes e bons avaliando-se arco de movimento e claudicação. Nossos resultados estão em concordância com a literatura, pois Kartenbender et al, em série de 35 pacientes (39 quadris) submetidos a osteotomia intertrocantérica, obtiveram $77 \%$ de resultados clínicos excelentes e bons, concluindo que este método, quando realizado de forma criteriosa, estaria indicado nos deslizamentos moderados e graves ${ }^{(13)}$.

Ao comparar dor e função com o critério radiológico, também em avaliação direta, pudemos observar, da mesma forma, esta discordância entre a análise subjetiva, que envolve diretamente a satisfação do paciente, e a análise objetiva.

Dos quatro (16\%) pacientes que apresentaram resultados ruins, em dois pode-se associar os maus resultados a erro no emprego da técnica e à indicação cirúrgica inadequada. Um apresentou material de síntese intra-articular, evoluindo para intensa degeneração articular, e o outro, condrólise pré-operatória, caracterizando má indicação cirúrgica. Este paciente já foi submetido a artroplastia total do quadril. Analisan- 
do-se os outros dois pacientes, classificados com resultado ruim, um apresentou condrólise pós-operatória, e outro, dor importante com sinais radiográficos de osteoartrose.

Schai et al, em série de 51 quadris tratados com osteotomia intertrocantérica, avaliados após 24 anos em média, descreveram que $55 \%$ dos quadris apresentavam-se sem alterações degenerativas e $28 \%$ mostravam sinais degenerativos apenas moderados, concluindo que seus resultados são superiores aos relatados para fixação in situ com mesmo grau de deslizamento(4).

\section{CONCLUSÃO}

A osteotomia intertrocantérica triplanar é boa opção no tratamento do EEPF maior de $30^{\circ}$, como demonstrado por nossos resultados, porém, a indicação deve ser criteriosa e é fundamental a realização de planejamento pré-operatório adequado, bem como técnica cirúrgica perfeita.

\section{REFERÊNCIAS}

1. Bellemans J, Fabry G, Molenaers G, Lammens J, Moens P. Slipped capital femoral epiphysis: a long-term follow-up, with special emphasis on the capacities for remodeling. J Pediatr Orthop B. 1996;5(3):151-7.

2. Engelhardt P. Spontanverlauf der Epiphyseolysis capitis femoris. Orthopade. 1994;23(3):195-9.

3. Clarke HJ, Wilkinson JA. Surgical treatment for severe slipping of the upper femoral epiphysis. J Bone Joint Surg Br. 1990; 72(5):854-8.

4. Schai PA, Exner GU, Hänsch O. Prevention of secondary coxarthrosis in slipped capital femoral epiphysis: a long-term follow-up study after corrective intertrochanteric osteotomy. J Pediatr Orthop B. 1996;5(3):135-43. Comment in: J Pediatr Orthop B. 2000;9(1):65-7.

5. Maussen JP, Rozing PM, Obermann WR. Intertrochanteric corrective osteotomy in slipped capital femoral epiphysis. A long-term follow-up study of 26 patients. Clin Orthop Relat Res. 1990;(259):100-10.

6. Barros JW, Oliveira EF, Barsam NHM, Fernandes CD, Miana LOA. Osteotomia do colo femoral no tratamento da epifisiólise grave. Rev Bras Ortop. 1995;30(7):489-92.
7. Fish JB. Cuneiform osteotomy of the femoral neck in the treatment of slipped capital femoral epiphysis. A follow-up note. J Bone Joint Surg Am. 1994;76(1):46-59.

8. Gage JR, Sundberg AB, Nolan DR, Sletten RG, Winter RB. Complications after cuneiform osteotomy for moderately or severely slipped capital femoral epiphysis. J Bone Joint Surg Am. 1978;60(2):157-65.

9. Hägglund G, Hansson LI, Ordberg G, Sandström S. Slipped capital femoral epiphysis in southern Sweden. Long-term results after femoral neck osteotomy. Clin Orthop Relat Res. 1986;(210):152-9.

10. Sampaio WF, Preuss AO. Osteotomia do colo no tratamento da epifisiólise femoral superior. Rev Bras Ortop. 1989;24(10): 348-54.

11. Southwick WO. Osteotomy through the lesser trochanter for slipped capital femoral epiphysis. J Bone Joint Surg Am. 1967; 49(5):807-35.

12. Cabral FP, Freitas E, Penedo JL, Rondinelli P, Carvalho PI, Chaparro JCA. Osteotomia tridimensional no tratamento do escorregamento epifisário superior do fêmur. Rev Bras Ortop. 1997;32(10):797-800.

13. Kartenbender K, Cordier W, Katthagen BD. Long-term followup study after corrective Imhäuser osteotomy for severe slipped capital femoral epiphysis. J Pediatr Orthop. 2000;20(6):74956

14. Kramer WG, Craig WA, Noel S. Compensating osteotomy at the base of the femoral neck for slipped capital femoral epiphysis. J Bone Joint Surg Am. 1976;58(6):796-800.

15. Carney BT, Weinstein SL, Noble J. Long-term follow-up of slipped capital femoral epiphysis. J Bone Joint Surg Am. 1991; 73(5):667-74.

16. Jerne R, Hansson G, Wallin J, Karlsson J. Long-term results after realignment operations for slipped upper femoral epiphysis. J Bone Joint Surg Br. 1996;78(5):745-50.

17. Aronson DD, Carlson WE. Slipped capital femoral epiphysis. A prospective study of fixation with a single screw. J Bone Joint Surg Am. 1992;74(6):810-9. Erratum in: J Bone Joint Surg Am. 1992;74(8):1274.

18. Jones JR, Paterson DC, Hillier TM, Foster BK. Remodelling after pinning for slipped capital femoral epiphysis. J Bone Joint Surg Br. 1990;72(4):568-73.

19. Lomelino RO, Mota Filho G, Schott PC, Mendes HM. Epifisiolistese proximal do fêmur: fixação "in situ”. Rev Bras Ortop. 1996;31(1):28-32.

20. Ireland J, Newman PH. Triplane osteotomy for severely slipped upper femoral epiphysis. J Bone Joint Surg Br. 1978;60-B(3): 390-3. 